Methods: Prospective observational study of a cohort of patients with sicca syndrome from a reference center. The diagnostic protocol (Schirmer's test, UWSF and minimally invasive MSGB) was applied in the same consultation. Demographic, clinical, analytical and histological data were reviewed.

Results: Over a period of 6 months, 48 patients with dry syndrome were analyzed, of which 39 women $(81.2 \%)$. The main suspicion was SjS (39), followed by sarcoidosis (3), IgG4-related disease (2) and other diagnoses (4). The mean age was $59.1 \pm 4.4$ years. Almost half $(45.8 \%)$ reported xerostomia and $41.6 \%$ xerophthalmia. Recurrent parotidomegaly was described in 6 patients (12.5\%) and arthralgias in 12 (25\%). Immunologically, 23 (47.9\%) presented anti-nuclear antibodies, $13(27.1 \%)$ anti-Ro, 4 (8.3\%) anti-La, 12 $(25 \%)$ rheumatoid factor and $15(31.2 \%)$ low C4. Schirmer test was positive in 32 patients $(66.7 \%)$, UWSF in $22(45.8 \%)$ and $9(18.8 \%)$ had a focus score $\geq 1$, although $16(33.3 \%)$ had focal lymphocytic sialadenitis in the MSGB. A total of $21(43,8 \%)$ patients were classified according to the $2016 \mathrm{ACR} /$ EULAR criteria. 12 (57.1\%) were seropositive SjS and 9 (42.9\%) seronegative SjS. MSGB sensitivity was $71 \%$ and specificity $96 \%$. Patient reported symptoms were unhelpful to differentiate SjS from other causes of dry syndrome. The number of protocols needed to diagnose a SjS was 2.28 (5.33 in seronegative $\mathrm{SjS}$ ). Complications associated with the procedure were low (1 of 48 ) and mild (self-limited paraesthesia). Patients with SjS, unlike those with dry syndrome of another etiology, had more anemia $(p<0.001)$, lymphopenia $(p=0.022)$, ESR $(p=0.030)$, beta-2 microglobulin $(p=0.011)$, ANA $(p<0.001)$, anti-ENA $(p=0.006)$, anti-Ro $(p<0.001)$, low C4 $(p<0.001)$ and hypergammaglobulinemia $(p=0.002)$.

Conclusion: Immunological and histological manifestations were more predictive than clinical ones to differentiate SjS from other causes of dry syndrome. MSGB is a simple, sensitive, specific and safe procedure. The application of the diagnostic protocol (Schirmer test, UWSF and MSGB) allowed to standardize the classification of SjS and increased the diagnosis of patients with seronegative SjS.

References:

[1] Ramos-Casals M, Brito-Zerón P, Bombardieri S On behalf of the EULARSjögren Syndrome Task Force Group, et al. EULAR recommendations for the management of Sjögren's syndrome with topical and systemic therapies. Annals of the Rheumatic Diseases 2020;79:3-18.

[2] Guellec D, Cornec D, Jousse-Joulin S, et al. Diagnostic value of labial minor salivary gland biopsy for Sjögren's syndrome: a systematic review. Autoimmun Rev. 2013;12(3):416-420.

Disclosure of Interests: None declared

DOI: 10.1136/annrheumdis-2020-eular.4921

\section{AB0397 LUPUS FATIGUE PROBLEMS IN THE RUSSIAN COHORT OF PATIENTS WITH SYSTEMIC LUPUS ERYTHEMATOSUS (RENAISSANCE).}

E. Aseeva ${ }^{1}$, S. Solovyev ${ }^{1}$, G. Koilubaeva ${ }^{2}$, N. Nikishina ${ }^{1}$, E. Nasonov ${ }^{1}$, A. Lila ${ }^{1}{ }^{1}$ V.A. Nasonova Research Institute of Rheumatology, Moscow, Russian Federation, Intensive Care Department, Moscow, Russian Federation; ${ }^{2}$ National Center for Cardiology and Internal Medicine named after Academician M. Mirrakhimov, Bishkek, Kyrgyz Republic, Rheumatology, Bishkek, Kyrgyzstan

\section{Background:}

Objectives: To determine the dependency of fatigue from SLE activity, irreversible organ damage and HRQOL in SLE patients of the Renaissance cohort.

Methods: 328 Russian SLE patients were enrolled in the study (M/F 30/298) who fulfilled SLICC 2012 criteria. The SLEDAI 2K activity, SLICC damage index, Facit Fatigue scale, and health related quality of life (HRQol) using the LupusQol questionnaire were evaluated.

Results: Based on the Facit Fatigue scale scores fatigue was verified in 148 (45\%) out of 328 patients with SLE. Following lupus fatigue status patients were divided into two groups - 148 and 168 patients respectively. The groups were perfectly matched in terms of age, duration of the disease, duration of GCs therapy, and damage scores (Table 1)

The SLEDAI $2 \mathrm{~K}$ activity scores were significantly higher in the group with lupus fatigue $-9.6 \pm 6.0$ vs $6.7 \pm 4.2(p=0.01)$ as compared to values in the group without fatigue, as well as the level of antibodies to DNA $(p=0.02), 110.2 \pm 34.2$ and $82.3 \pm 20.5$, respectively.

There was a significant decrease in $\mathrm{HRQ}$ L in patients with lupus fatigue based on scores in all eight scales of the questionnaire $(p=0.00)$

Table 1. Comparative analysis of 328 SLE patients with and without lupus fatigue

\begin{tabular}{lccc}
\hline $\begin{array}{l}\text { Characteristics } \\
\text { mean (SD) }\end{array}$ & $\begin{array}{c}\text { Patients with } \\
\text { lupus fatigue } \\
(\mathbf{n}=\mathbf{1 4 8})\end{array}$ & $\begin{array}{c}\text { Patients without } \\
\text { lupus fatigue } \\
(\mathbf{n}=\mathbf{1 6 8})\end{array}$ & $\begin{array}{c}\mathbf{P} \\
\text { (Mann- } \\
\text { Whitney) }\end{array}$ \\
\hline Age in years & $35,31 \pm 12,03$ & $33,68 \pm 10,68$ & 0,13 \\
Disease durationin in years & $9,57 \pm 9,47$ & $10,1 \pm 9,17$ & 0,6 \\
Duration of glucocorticoid therapy in month & $72,81 \pm 7158$ & $79,43 \pm 75,98$ & 0,6 \\
SLICC Dal, score & $1,55 \pm 1,37$ & $1,69 \pm 1,23$ & 0,44 \\
C3 Mulml & $0,88 \pm 0,33$ & $0,87 \pm 0,31$ & 0,75 \\
C4 Mulml & $0,15 \pm 0,12$ & $0,16 \pm 0,14$ & 0,36 \\
ANF, hep2 & $641 \pm 453$ & $550 \pm 402$ & 0,4 \\
Fatigue scores by the Facit fatigue scale & $25,16 \pm 6,58$ & $42,43 \pm 4,56$ & 0,000 \\
\hline
\end{tabular}

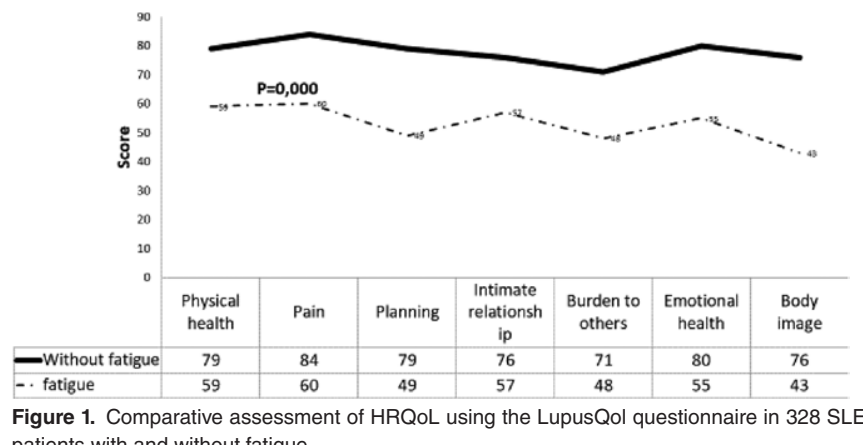

patients with and without fatigue.

Conclusion: Almost half (45\%) SLE patients in the Russian cohort experience fatigue. It is associated with disease activity on the SLEDAI $2 \mathrm{k}$ scale and high levels of antibodies to DNA. All patients experiencing lupus fatigue have significantly worse HRQoL based on scores in all eight scales of the questionnaire LupusQol.

Disclosure of Interests: None declared

DOI: 10.1136/annrheumdis-2020-eular.3167

\section{$\mathrm{AB0398}$}

TNF-LIKE WEAK INDUCER OF APOPTOSIS / FGF INDUCIBLE MOLECULE 14 PATHWAY IN UNTREATED LUPUS NEPHRITIS: SERUM OR URINE TWEAK LEVELS MORE ACCURATE IN RELATION TO RENAL ACTIVITY?

M. Bekhit ${ }^{1}$, N. Abaza ${ }^{1}$, N. Kamel ${ }^{1}$, M. Ossman ${ }^{1}$, S. Saad El Din ${ }^{2} .{ }^{1}$ Ain Shams University, Rheumatology, Cairo, Egypt; ${ }^{2}$ Ain Shams University, Pathology, Cairo, Egypt

Background: Lupus nephritis (LN) is one of the most serious manifestations of SLE, affecting $70 \%$ of patients and the most critical predictor of morbidity and mortality of the disease [1].Tumor necrosis factor-like weak inducer of apoptosis/ fibroblast growth factor inducible molecule 14 (TWEAK/Fn14) activation is involved in various pathological processes that occur locally in kidneys, facilitating the pathogenesis of LN [2].

Objectives: To assess serum and urine TWEAK levels as well as renal Fn14 expression in newly diagnosed patients with $\mathrm{LN}$ and its correlation to disease activity.

Methods: The present study included 30 selected newly diagnosed previously untreated SLE patients divided into 2 groups; 15 patients with LN and 15 without $L N$ as well as 30 age and sex matched healthy subjects who served as control group. Written consent was obtained from all patients and 
controls after a full explanation of the study. Clinical assessment of disease activity by SLE Disease activity index (SLEDAI) [3]. Lupus nephritis was assessed clinically with the renal SLE disease activity index (rSLEDAI).Indicated renal biopsies were taken from the patients with LN and were classified according to the International Society of Nephrology and the Renal Pathology Society (ISN/RPS) classification [4].Serum and urinary levels of TWEAK were measured for the patients and controls using enzyme-linked immunosorbent assay (ELISA). Fn 14 was examined in renal biopsies from LN group by immunohistochemistry.

Results: A significantly higher UTWEAK level on comparing SLE patients with LN to those without $L N$ and controls $(F=149.2, P<0.001)$. UTWEAK had a highly significant positive correlation with, proteinuria $(r=0.755, P<0.001)$, a significant positive correlation with SLEDAI and rSLEADI $(r=0.217, P<0.037)(r$ $=0.476, P<0.024)$ respectively. UTWEAK had a significant negative correlation with anti-dsDNA titres, C3 and C4 $(r=-0.579, P<0.008)(r=-0.456, P<0.011)$ $(r=-0.552, P<0.002)$. Although sTWEAK level was higher in SLE patients than controls, it was not found to be associated with the presence of $L N(F=4.963$, $\mathrm{P}=0.012)$. Fn14 expression was detected in glomerular and tubular cells in LN patients.
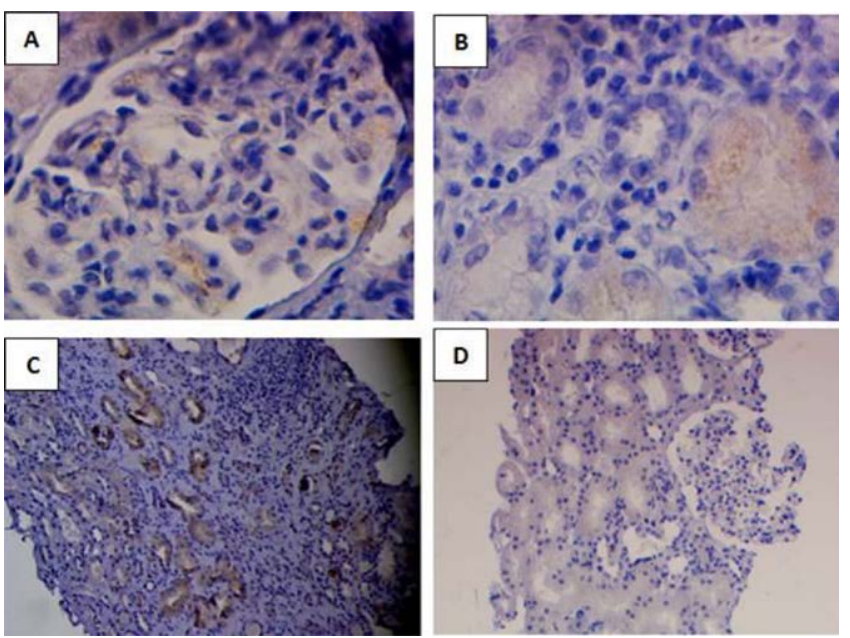

Expression of Fn14 in renal biopsies from LN patients was examined. Immunohistochemistry for Fn14 was detected in (A) glomerular endothelial cells, $(B, C)$ some specimens showed moderate and intense staining for Fn14 in renal tubular cells. In the controls [renal biopsies from patients with renal cell carcinoma (D)], there was very slight staining for Fn14 in renal tubular cells.

Conclusion: Urinary TWEAK is a specific and sensitive biomarker for detection of active LN in newly diagnosed untreated SLE patients.

References:

[1] Kwok SK and Tsokos GC. New insights into the role of renal resident cells in the pathogenesis of lupus nephritis. Korean J Intern Med 2018; 33 (2): 284-289.

[2] Xia Y, Herlitz LC, Gindea S, Wen J, Pawar RD, Misharin A, et al. Deficiency of Fibroblast Growth Factor-Inducible 14 (Fn14) Preserves the Filtration Barrier and Ameliorates Lupus Nephritis. J Am Soc Nephrol 2015; 26(5):1053-1070.

[3] Bombardier C, Gladman DD, Urowitz MB, Caron D, Chang CH, Derivation of the SLEDAI. A disease activity index of lupus patients. Arthritis Rheum 1992; 35: $630-40$.

[4] Weening JJ, D'Agati VD, Schwartz MM, Seshan SV, Alpers CE, Appel GB, et al. The classification of glomerulonephritis in systemic lupus erythematosus revisited. J Am Soc Nephrol 2004; 15(2): 241-250.

Disclosure of Interests: None declared

DOI: 10.1136/annrheumdis-2020-eular.2196

\section{AB0399 \\ SYSTEMIC LUPUS ERYTHEMATOSUS AND OPPORTUNISTIC INFECTIONS: PREVALENCE, FEATURES OF CLINICAL SYMPTOMS}

B. Belov ${ }^{1}$, O. Egorova ${ }^{1}$, D. Movsisyan ${ }^{2} .{ }^{1}$ V.A. Nasonova Research Institute of Rheumatology, Moscow, Russian Federation; ${ }^{2}$ Pirogov Russian National Research Medical University, Moscow, Russian Federation
Background: Modern therapy for systemic lupus erythematosus (SLE) is associated with long-term treatment with cytotoxic drugs which is often accompanied by activation of comorbid infection, including viral infection.

Objectives: to identify prevalence and specific characteristics of the clinical symptoms of co-infection in SLE.

Methods: 67 patients with SLE were examined, mainly middle-aged women (62) of $33.5 \pm 8.1$ years old with the disease duration of 1 to 7 years who received "basic therapy". All patients were examined by ELISA for the presence of Herpesviridae viruses.

Results: In 67 patients, ELISA revealed anti-viral antibodies IgG to CMV $(86.5 \%)$ and VCA IgG of EBV $(56.7 \%)$, somewhat less frequently than HSV-1 IgG (38.8\%) and HSV-2 (26. $8 \%$ ). Active CMV and EBV infections were diagnosed in $26(38.8 \%)$ and $19(28.3 \%)$ patients, respectively; moreover, in 11 patients a combination of production of antibodies of the IgM CMV and EA IgG EBV class was revealed. Analysis of anamnestic data and serological examination allowed us to distinguish 3 groups of patients: group I- 35 patients with a viral infection, including 9 with the infection of a mixed viral and bacterial nature; Group II - 14 patients with bacterial infection and Group III - 18 patients without viral and bacterial complications. Analysis of clinical symptoms showed that there is a definite correlation of high titers of antibodies to CMV and EBV with symptoms such as fever $(p<0.01, r=0.74)$, polyarthralgia $(p<0.02, r=0.46)$ lymphadenopathy $(p<0.01, r=0.74)$, carditis $(p<0.05, r=0.42)$, hepatomegaly $(p<0.05, r=0.62)$, central nervous system damage $(p<0.02, r=0.46)$, migratory erythematous/hemorrhagic rash $(p<0.05, r=0.58)$, urinary syndrome $(p<0.02, r=0.41)$, anti-dsDNA $(p<0.01$, $r=0.82)$, ANA $(p<0.02, r=0.74)$; cryoprecipitins $(p<0.01, r=0.45)$. Although there were similar clinical manifestations, the presence of CMV and EBV had some organ specificity. Thus, damage to the central nervous system, joints (polyarthralgia) and liver was more common in patients with CMV, and lymphadenopathy and erythematous/hemorrhagic rash - in patients with EBV.

Conclusion: In SLE a comorbid viral infection contributes to clinical picture with a lingering remitting inflammatory process, as well as insufficient effectiveness from corticosteroid and immunosuppressive therapy.

Disclosure of Interests: None declared

DOI: 10.1136/annrheumdis-2020-eular.1444

\section{AB0400 PATIENT-REPORTED OUTCOME MEASURES IN SWEDISH PATIENTS WITH RECENT-ONSET SLE VERSUS RA IN THE FIRST 60 MONTHS AFTER DIAGNOSIS}

M. Bjork ${ }^{1}$, I. Thyberg ${ }^{1}$, A. Kastbom ${ }^{1}$, R. Heijke ${ }^{1}$, L. Mcdonald ${ }^{2}$, E. Alemao ${ }^{3}$ C. Sjowall ${ }^{1}{ }^{1}$ Linköping University, Linköping, Sweden; ${ }^{2}$ Bristol-Myers Squibb, Uxbridge, United Kingdom; ${ }^{3}$ Bristol-Myers Squibb, Princeton, United States of America

Background: Patient (pt)-reported outcome measures (PROMs) provide information on a pt's own perception of disease impact, helping to provide a global perspective of disease when combined with conventional physician assessments. There is a discrepancy in PROMs between pts with rheumatoid arthritis (RA) and those with systemic lupus erythematosus (SLE); improvements in PROMs are often seen within the first year after diagnosis among pts with RA, but not SLE. ${ }^{2}$ Whether this discrepancy persists during subsequent years is unknown

Objectives: To compare changes in PROMs among pts with SLE versus RA within the first 60 months after diagnosis.

Methods: Pts with SLE with no prior organ damage were consecutively enrolled (2010-2015) from the Clinical Lupus Register in Northeastern Gothia and met the $\geq 4$ of the 1982 American College of Rheumatology (ACR) and/or the 2012 Systemic Lupus International Collaborating Clinics classification criteria. Pts with RA were included from the observational 2nd Management of Early Intervention in RA cohort (TIRA-2; 2006-2009), ${ }^{3}$ which enrolled pts with recent-onset RA; $84 \%$ of patients fulfilled the 1987 ACR criteria. Pts in both cohorts had symptoms for $<12$ months prior to diagnosis/inclusion and were treated according to Swedish guidelines. Pts with SLE or RA were followed prospectively after diagnosis. PROMs (quality of life: EuroQoL-5 Dimensions [EQ-5D]; activity limitations: Health Assessment Questionnaire [HAQ]; pain, fatigue, and well-being: Visual Analogue Scale $0-100 \mathrm{~mm}$ ) were collected at Months 0 (inclusion), 6, 12, 24, 36, 48, and 60. HAQ, pain, fatigue, well-being: higher scores indicate greater severity; EQ-5D: lower scores indicate greater severity. 